PROCEEDINGS OF THE

AMERICAN MATHEMATICAL SOCIETY

Volume 138, Number 2, February 2010, Pages 687-693

S 0002-9939(09)10090-4

Article electronically published on October 2, 2009

\title{
GROUND STATES OF NONLINEAR SCHRÖDINGER SYSTEMS
}

\author{
JINYONG CHANG AND ZHAOLI LIU
}

(Communicated by Yingfei Yi)

\begin{abstract}
This paper concerns the existence of positive radial ground states of the time-independent Schrödinger system

$$
\begin{cases}-\Delta u_{1}+\lambda_{1} u_{1}=\mu_{1} u_{1}^{3}+\beta u_{2}^{2} u_{1}, & \text { in } \mathbb{R}^{n}, \\ -\Delta u_{2}+\lambda_{2} u_{2}=\mu_{2} u_{2}^{3}+\beta u_{1}^{2} u_{2}, & \text { in } \mathbb{R}^{n}, \\ u_{1}(x) \rightarrow 0, \quad u_{2}(x) \rightarrow 0, & \text { as }|x| \rightarrow \infty\end{cases}
$$

where $n=1,2,3, \lambda_{j}>0$ and $\mu_{j}>0$ for $j=1,2$, and $\beta>0$. A result from Sirakov, Comm. Math. Phys. 271 (2007), 199-221, is improved.
\end{abstract}

Consider the time-independent Schrödinger system

$$
\begin{cases}-\Delta u_{1}+\lambda_{1} u_{1}=\mu_{1} u_{1}^{3}+\beta u_{2}^{2} u_{1}, & \text { in } \mathbb{R}^{n}, \\ -\Delta u_{2}+\lambda_{2} u_{2}=\mu_{2} u_{2}^{3}+\beta u_{1}^{2} u_{2}, & \text { in } \mathbb{R}^{n}, \\ u_{1}(x) \rightarrow 0, \quad u_{2}(x) \rightarrow 0, & \text { as }|x| \rightarrow \infty,\end{cases}
$$

where $n=1,2,3, \lambda_{j}>0$ and $\mu_{j}>0$ for $j=1,2$, and $\beta>0$. Solutions $\left(u_{1}(x), u_{2}(x)\right)$ of (11) correspond to standing wave solutions $\left(e^{i \lambda_{1} t} u_{1}(x), e^{i \lambda_{2} t} u_{2}(x)\right)$ of the timedependent system of 2 coupled nonlinear Schrödinger equations

$$
\begin{cases}-i \frac{\partial}{\partial t} \Phi_{1}=\Delta \Phi_{1}+\mu_{1}\left|\Phi_{1}\right|^{2} \Phi_{1}+\beta\left|\Phi_{2}\right|^{2} \Phi_{1}, & \text { in } \mathbb{R}^{n}, \\ -i \frac{\partial}{\partial t} \Phi_{2}=\Delta \Phi_{2}+\mu_{2}\left|\Phi_{2}\right|^{2} \Phi_{2}+\beta\left|\Phi_{1}\right|^{2} \Phi_{2}, & \text { in } \mathbb{R}^{n}, \\ \Phi_{1}(x, t) \rightarrow 0, \quad \Phi_{2}(x, t) \rightarrow 0, & \text { as }|x| \rightarrow \infty\end{cases}
$$

The system (2) stems from many physical problems, especially in nonlinear optics and in the Hartree-Fock theory for Bose-Einstein condensates; see, for example, [1, 5, 8, 9, 10, 11, 12, 20, 23.

Recently, (11) has attracted tremendous attention and has been studied extensively from the point of view of physics (see 11, 10, 11 for instance) as well as mathematics (see [2, 3, 4, 13, 14, 15, 16, 18, 19, 21, 22, 24, 25]). Solutions of (1) correspond to critical points of the energy functional

$$
E(\vec{u})=\frac{1}{2} \int_{\mathbb{R}^{n}}\left(\left|\nabla u_{1}\right|^{2}+\lambda_{1} u_{1}^{2}+\left|\nabla u_{2}\right|^{2}+\lambda_{2} u_{2}^{2}\right)-\frac{1}{4} \int_{\mathbb{R}^{n}}\left(\mu_{1} u_{1}^{4}+2 \beta u_{1}^{2} u_{2}^{2}+\mu_{2} u_{2}^{4}\right),
$$

defined for $\vec{u}=\left(u_{1}, u_{2}\right) \in\left(H^{1}\left(\mathbb{R}^{n}\right)\right)^{2}$. A solution $\left(u_{1}, u_{2}\right)$ of (1) is called a nontrivial solution if $u_{1} \neq 0$ and $u_{2} \neq 0$, a semitrivial solution if either $u_{1} \neq 0$ or $u_{2} \neq 0$, a positive solution if $u_{1}>0$ and $u_{2}>0$, and a semipositive solution if $u_{1} \geq 0$ and

Received by the editors March 24, 2009, and, in revised form, June 19, 2009.

2000 Mathematics Subject Classification. Primary 35J10, 35J50, 58E05.

Key words and phrases. Schrödinger system, nontrivial ground state, Morse index.

This work was supported by NSFC (10825106). 
$u_{2} \geq 0$ and if either $u_{1} \neq 0$ or $u_{2} \neq 0$. A solution is said to be a ground state if it has the least energy among all semitrivial solutions.

Let $H_{r}^{1}\left(\mathbb{R}^{n}\right)$ consist of all radial functions in $H^{1}\left(\mathbb{R}^{n}\right)$. Denote $X=\left(H^{1}\left(\mathbb{R}^{n}\right)\right)^{2}$ and $X_{r}=\left(H_{r}^{1}\left(\mathbb{R}^{n}\right)\right)^{2}$. It was proved in [4] that (10) has a semipositive radial ground state which is of mountain pass type and has Morse index 1 when considered as the critical point of $E$ on $X$ and on $X_{r}$. As indicated in [4, a semipositive ground state may only be semipositive and therefore there does not exist a positive ground state, and this is the case if, for example, $\lambda_{1} \leq \lambda_{2}$ and $\mu_{1} \geq \beta \geq \mu_{2}$ and at least one inequality among the three is strict. Therefore, a natural problem is when (11) has a positive ground state. In the case $\lambda_{1}=\lambda_{2}$, there is a complete answer from [4] which states that (11) has a positive ground state if and only if $\beta>\max \left\{\mu_{1}, \mu_{2}\right\}$ or $\beta=\mu_{1}=\mu_{2}$. Explicit positive ground states can be obtained in this case. According to [22, $\left(u_{1}, u_{2}\right)$ with $u_{1}$ and $u_{2}$ defined by

$$
u_{1}=\sqrt{\frac{\lambda\left(\beta-\mu_{2}\right)}{\beta^{2}-\mu_{1} \mu_{2}}} w(\sqrt{\lambda} \cdot) \text { and } u_{2}=\sqrt{\frac{\lambda\left(\beta-\mu_{1}\right)}{\beta^{2}-\mu_{1} \mu_{2}}} w(\sqrt{\lambda} \cdot),
$$

where $w$ is the unique positive radial solution of

$$
-\Delta w+w=w^{3} \text { in } \mathbb{R}^{n}, \quad w(x) \rightarrow 0 \text { as }|x| \rightarrow \infty,
$$

is a positive ground state of (11) if $\lambda_{1}=\lambda_{2}$ (denoted by $\lambda$ ) and $\beta>\max \left\{\mu_{1}, \mu_{2}\right\}$. Also, a direct computation shows that

$$
(\cos \theta \sqrt{\lambda / \beta} w(\sqrt{\lambda} \cdot), \sin \theta \sqrt{\lambda / \beta} w(\sqrt{\lambda} \cdot)), \quad \theta \in(0, \pi / 2)
$$

are positive ground states of (11) if $\lambda_{1}=\lambda_{2}$ (denoted by $\lambda$ ) and $\beta=\mu_{1}=\mu_{2}$. Results similar to [4] were proved in [6], using the bifurcation theorem.

For $\lambda_{1} \neq \lambda_{2}$ the problem of whether (11) has a positive ground state remains largely open. In what follows, we shall always assume $\lambda_{2} \geq \lambda_{1}$ without introducing any essential restriction. In this general case, Bartsch and Wang [4] first proved that there is some $b>0$ such that the ground state solution for (11) is positive if $\beta>b$. But no information on how large $b$ is was given. Assuming $\lambda_{1}=1$ and $\lambda:=\lambda_{2} \geq 1$, Sirakov (see [22, Theorem 2(iv) and Section 3.4]) proved that if

$$
\beta>\max \left\{\lambda \mu_{1}, \lambda^{\frac{n}{2}-1} \mu_{2}\right\},
$$

then (11) has a positive ground state. The aim of the present paper is to improve this result of Sirakov.

The argument of this paper is motivated by those in [4]. We shall distinguish nontrivial solutions from those which are only semitrivial (that is, solutions with only one component being nonzero) by comparing the Morse indices of them.

To state our result, we first introduce the notation

$$
C_{1}=\int_{\mathbb{R}^{n}} w^{2}(x) d x, \quad C_{2}=\int_{\mathbb{R}^{n}} w^{4}(x) d x .
$$

Theorem 1. Assume $\lambda_{1} \leq \lambda_{2}$. If

$$
\beta>\max \left\{\mu_{1}+\frac{\left(\lambda_{2}-\lambda_{1}\right) C_{1}}{\lambda_{1} C_{2}} \mu_{1}, \mu_{2}-\frac{\left(\lambda_{2}-\lambda_{1}\right) C_{1}}{\lambda_{2} C_{2}} \mu_{2}\right\},
$$

then (11) has a positive radial ground state. 
Remark 2. a) We first compare Theorem 1 with [22, Theorem 2(iv)] in the two and three dimensional cases. Since $w$ is a positive solution of (3), we see that $0<C_{1}<C_{2}$ and therefore

$$
\frac{\lambda_{2}}{\lambda_{1}} \mu_{1}>\mu_{1}+\frac{\left(\lambda_{2}-\lambda_{1}\right) C_{1}}{\lambda_{1} C_{2}} \mu_{1} .
$$

Since clearly, for $n=2,3$,

$$
\left(\frac{\lambda_{2}}{\lambda_{1}}\right)^{\frac{n}{2}-1} \mu_{2}>\mu_{2}-\frac{\left(\lambda_{2}-\lambda_{1}\right) C_{1}}{\lambda_{2} C_{2}} \mu_{2}
$$

the assumption (5) is weaker than (4). Thus our theorem improves [22, Theorem 2(iv)]. Note that other sufficient assumptions were also given in [22] (see [Proposition 3.7] therein) to guarantee existence of a positive radial ground state of (11). Nevertheless, those assumptions involve heavy notation and were described with quite complex inequalities, which are not easy to verify.

b) It seems that (15) is only a sufficient condition for (1) to have a positive radial ground state in the case $\lambda_{1}<\lambda_{2}$. But if $\lambda_{1}=\lambda_{2}$, then (5) reduces to

$$
\beta>\max \left\{\mu_{1}, \mu_{2}\right\} \text {, }
$$

and in this case (5) is necessary for (10) to have a positive radial ground state provided that $\mu_{1} \neq \mu_{2}$, according to [4].

c) The argument provided here cannot be generalized to systems consisting of more than 2 equations. By comparing energies, a positive radial ground state for systems consisting of $N(N \geq 2)$ equations was recently obtained in [17.

Proof of Theorem 1. Denote

$$
w_{j}(x)=\sqrt{\frac{\lambda_{j}}{\mu_{j}}} w\left(\sqrt{\lambda_{j}} x\right), \quad j=1,2 .
$$

Then, for $j=1,2, w_{j}$ is the unique solution of the equation

$$
\left\{\begin{array}{l}
-\Delta w+\lambda_{j} w=\mu_{j} w^{3} \text { and } w>0 \text { in } \mathbb{R}^{n}, \\
w(0)=\max w(x), \quad w(x) \rightarrow 0 \text { as }|x| \rightarrow \infty .
\end{array}\right.
$$

Set $U_{1}=\left(w_{1}, 0\right)$ and $U_{2}=\left(0, w_{2}\right)$. Then $U_{1}$ and $U_{2}$ are the only two semipositive radial solutions of (1) with only one component being nonzero.

It has been proved in [4] that (1) has a semipositive radial ground state $\vec{u} \in X_{r}$ and its Morse index is 1 . In order to prove that this ground state solution is positive, it suffices to prove that $U_{1}$ and $U_{2}$ have Morse indices at least 2. We now estimate the Morse indices of $U_{1}$ and $U_{2}$. For any $\left(\phi_{1}, \phi_{2}\right) \in\left(H^{1}\left(\mathbb{R}^{n}\right)\right)^{2}$, we have

$$
\begin{aligned}
E^{\prime \prime}\left(U_{1}\right) & {\left[\left(\phi_{1}, \phi_{2}\right),\left(\phi_{1}, \phi_{2}\right)\right] } \\
= & \int_{\mathbb{R}^{n}}\left(\left|\nabla \phi_{1}\right|^{2}+\lambda_{1} \phi_{1}^{2}+\left|\nabla \phi_{2}\right|^{2}+\lambda_{2} \phi_{2}^{2}\right)-\int_{\mathbb{R}^{n}}\left(3 \mu_{1} w_{1}^{2} \phi_{1}^{2}+\beta w_{1}^{2} \phi_{2}^{2}\right) .
\end{aligned}
$$

Thus, for any $t_{1}, t_{2} \in \mathbb{R}$,

$$
\begin{aligned}
E^{\prime \prime}\left(U_{1}\right) & {\left[\left(t_{1} w_{1}, t_{2} w_{1}\right),\left(t_{1} w_{1}, t_{2} w_{1}\right)\right] } \\
& =t_{1}^{2} \int_{\mathbb{R}^{n}}\left(\left|\nabla w_{1}\right|^{2}+\lambda_{1} w_{1}^{2}-3 \mu_{1} w_{1}^{4}\right)+t_{2}^{2} \int_{\mathbb{R}^{n}}\left(\left|\nabla w_{1}\right|^{2}+\lambda_{2} w_{1}^{2}-\beta w_{1}^{4}\right) \\
& =t_{1}^{2} \int_{\mathbb{R}^{n}}\left(-2 \mu_{1} w_{1}^{4}\right)+t_{2}^{2} \int_{\mathbb{R}^{n}}\left(\left(\mu_{1}-\beta\right) w_{1}^{4}+\left(\lambda_{2}-\lambda_{1}\right) w_{1}^{2}\right) .
\end{aligned}
$$


Since

$$
\begin{aligned}
\int_{\mathbb{R}^{n}}\left(\left(\mu_{1}-\beta\right) w_{1}^{4}+\left(\lambda_{2}-\lambda_{1}\right) w_{1}^{2}\right) & =-\frac{\left(\beta-\mu_{1}\right) \lambda_{1}^{2-\frac{n}{2}} C_{2}}{\mu_{1}^{2}}+\frac{\left(\lambda_{2}-\lambda_{1}\right) \lambda_{1}^{1-\frac{n}{2}} C_{1}}{\mu_{1}} \\
& =\frac{\lambda_{1}^{2-\frac{n}{2}} C_{2}}{\mu_{1}^{2}}\left[-\beta+\mu_{1}+\frac{\left(\lambda_{2}-\lambda_{1}\right) C_{1}}{\lambda_{1} C_{2}} \mu_{1}\right],
\end{aligned}
$$

we see that if

$$
\beta>\mu_{1}+\frac{\left(\lambda_{2}-\lambda_{1}\right) C_{1}}{\lambda_{1} C_{2}} \mu_{1},
$$

then, for any $\left(t_{1}, t_{2}\right) \in \mathbb{R}^{2} \backslash\{(0,0)\}$,

$$
E^{\prime \prime}\left(U_{1}\right)\left[\left(t_{1} w_{1}, t_{2} w_{1}\right),\left(t_{1} w_{1}, t_{2} w_{1}\right)\right]<0 .
$$

Therefore, if (6) holds, then the Morse index of $U_{1}$ is at least 2. Similarly, for any $\left(\phi_{1}, \phi_{2}\right) \in\left(H^{1}\left(\mathbb{R}^{n}\right)\right)^{2}$, we have

$$
\begin{aligned}
E^{\prime \prime}\left(U_{2}\right) & {\left[\left(\phi_{1}, \phi_{2}\right),\left(\phi_{1}, \phi_{2}\right)\right] } \\
= & \int_{\mathbb{R}^{n}}\left(\left|\nabla \phi_{1}\right|^{2}+\lambda_{1} \phi_{1}^{2}+\left|\nabla \phi_{2}\right|^{2}+\lambda_{2} \phi_{2}^{2}\right)-\int_{\mathbb{R}^{n}}\left(3 \mu_{2} w_{2}^{2} \phi_{2}^{2}+\beta w_{2}^{2} \phi_{1}^{2}\right) .
\end{aligned}
$$

Choosing $\left(\phi_{1}, \phi_{2}\right)=\left(t_{1} w_{2}, t_{2} w_{2}\right)$, one then obtains

$$
\begin{aligned}
& E^{\prime \prime}\left(U_{2}\right)\left[\left(t_{1} w_{2}, t_{2} w_{2}\right),\left(t_{1} w_{2}, t_{2} w_{2}\right)\right] \\
& \quad=t_{1}^{2} \int_{\mathbb{R}^{n}}\left(\left|\nabla w_{2}\right|^{2}+\lambda_{1} w_{2}^{2}-\beta w_{2}^{4}\right)+t_{2}^{2} \int_{\mathbb{R}^{n}}\left(\left|\nabla w_{2}\right|^{2}+\lambda_{2} w_{2}^{2}-3 \mu_{2} w_{2}^{4}\right) \\
& \quad=t_{1}^{2} \int_{\mathbb{R}^{n}}\left(\left(\mu_{2}-\beta\right) w_{2}^{4}+\left(\lambda_{1}-\lambda_{2}\right) w_{2}^{2}\right)+t_{2}^{2} \int_{\mathbb{R}^{n}}\left(-2 \mu_{2} w_{2}^{4}\right) .
\end{aligned}
$$

But

$$
\begin{aligned}
\int_{\mathbb{R}^{n}}\left(\left(\mu_{2}-\beta\right) w_{2}^{4}+\left(\lambda_{1}-\lambda_{2}\right) w_{2}^{2}\right) & =\frac{\lambda_{2}^{2-\frac{n}{2}}\left(\mu_{2}-\beta\right) C_{2}}{\mu_{2}^{2}}+\frac{\lambda_{2}^{1-\frac{n}{2}}\left(\lambda_{1}-\lambda_{2}\right) C_{1}}{\mu_{2}} \\
& =\frac{\lambda_{2}^{2-\frac{n}{2}} C_{2}}{\mu_{2}^{2}}\left[\mu_{2}-\beta-\frac{\left(\lambda_{2}-\lambda_{1}\right) C_{1}}{\lambda_{2} C_{2}} \mu_{2}\right] .
\end{aligned}
$$

Therefore, if

$$
\beta>\mu_{2}-\frac{C_{1}\left(\lambda_{2}-\lambda_{1}\right)}{C_{2} \lambda_{2}} \mu_{2},
$$

then, for any $\left(t_{1}, t_{2}\right) \in \mathbb{R}^{2} \backslash\{(0,0)\}$,

$$
E^{\prime \prime}\left(U_{2}\right)\left[\left(t_{1} w_{2}, t_{2} w_{2}\right),\left(t_{1} w_{2}, t_{2} w_{2}\right)\right]<0,
$$

which implies that if (7) holds, then the Morse index of $U_{2}$ is at least 2. Now if $\beta$ satisfies both (6) and (77), then the Morse indices of $U_{1}$ and $U_{2}$ are at least 2. Since $U_{1}$ and $U_{2}$ are the only two semipositive radial solutions of (11) with only one component being nonzero and since the semipositive radial ground state obtained in [4] has Morse index 1, the semipositive radial ground state is positive.

Remark 3. In estimating the lower bound of the Morse index of $E$ at $U_{1}$, we have used the two-dimensional subspace spanned by $\left(w_{1}, 0\right)$ and $\left(0, w_{1}\right)$ as a test subspace, other than $\operatorname{span}\left\{\left(w_{2}, 0\right),\left(0, w_{1}\right)\right\}$ or $\operatorname{span}\left\{\left(w_{1}, 0\right),\left(0, w_{2}\right)\right\}$ or $\operatorname{span}\left\{\left(w_{2}, 0\right)\right.$, $\left.\left(0, w_{2}\right)\right\}$. The reason is that the subspace $\operatorname{span}\left\{\left(w_{1}, 0\right),\left(0, w_{1}\right)\right\}$ among the four 
yields the most transparent estimate. All the other subspaces do not give an estimate which can be described as simply as (5). The same remark applies to the argument of estimating the lower bound of the Morse index of $E$ at $U_{2}$. Nevertheless, if we use all four subspaces as test subspaces to estimate the Morse indices of $E$ at $U_{1}$ and $U_{2}$, then we obtain the following result.

Theorem 4. Assume $\lambda_{1} \leq \lambda_{2}$. If

$$
\beta>\max \left\{m_{1}\left(n, \lambda_{1}, \lambda_{2}\right) \mu_{1}, m_{2}\left(n, \lambda_{1}, \lambda_{2}\right) \mu_{2}\right\},
$$

where

$$
\begin{aligned}
& m_{1}\left(n, \lambda_{1}, \lambda_{2}\right)=\min \left\{1+\left(\lambda_{2}-\lambda_{1}\right) \lambda_{1}^{-1} C_{1} C_{2}^{-1}, \lambda_{2}^{1-\frac{n}{2}} \lambda_{1}^{-1} C_{2} C^{-1}\left(\lambda_{1}, \lambda_{2}\right)\right\}, \\
& m_{2}\left(n, \lambda_{1}, \lambda_{2}\right)=\min \left\{1-\left(\lambda_{2}-\lambda_{1}\right) \lambda_{2}^{-1} C_{1} C_{2}^{-1}, \lambda_{1}^{1-\frac{n}{2}} \lambda_{2}^{-1} C_{2} C^{-1}\left(\lambda_{1}, \lambda_{2}\right)\right\},
\end{aligned}
$$

and

$$
C\left(\lambda_{1}, \lambda_{2}\right)=\int_{\mathbb{R}^{n}} w^{2}\left(\sqrt{\lambda_{1}} x\right) w^{2}\left(\sqrt{\lambda_{2}} x\right) d x
$$

then (11) has a positive radial ground state.

Remark 5. a) One may think of giving a comparison between the numbers $1+$ $\left(\lambda_{2}-\lambda_{1}\right) \lambda_{1}^{-1} C_{1} C_{2}^{-1}$ and $\lambda_{2}^{1-\frac{n}{2}} \lambda_{1}^{-1} C_{2} C^{-1}\left(\lambda_{1}, \lambda_{2}\right)$ as well as a comparison between $1-\left(\lambda_{2}-\lambda_{1}\right) \lambda_{2}^{-1} C_{1} C_{2}^{-1}$ and $\lambda_{1}^{1-\frac{n}{2}} \lambda_{2}^{-1} C_{2} C^{-1}\left(\lambda_{1}, \lambda_{2}\right)$. We do not know how to do this at the present stage. Therefore, we do not know if the result in Theorem 4 is better than the result in Theorem 1 .

b) But in the $n=1$ dimensional case, $w$ has an explicit expression:

$$
w(x)=\frac{2 \sqrt{2}}{e^{x}+e^{-x}} .
$$

Using this expression, we easily see that $C_{1}=4$ and $C_{2}=\frac{16}{3}$. If, for example, $\lambda_{2}=4 \lambda_{1}$ or $\lambda_{2}=9 \lambda_{1}$, then after a long but elementary calculation, we obtain

$$
C\left(\lambda_{1}, 4 \lambda_{1}\right)=\frac{4(4-\pi)}{\sqrt{\lambda_{1}}}, \quad C\left(\lambda_{1}, 9 \lambda_{1}\right)=\frac{16}{3 \sqrt{\lambda_{1}}}-\frac{128 \pi}{81 \sqrt{3 \lambda_{1}}} .
$$

Then the four numbers mentioned in a) can be estimated in the following table.

\begin{tabular}{|c|c|c|}
\hline & $\lambda_{2}=4 \lambda_{1}$ & $\lambda_{2}=9 \lambda_{1}$ \\
\hline $1+\left(\lambda_{2}-\lambda_{1}\right) \lambda_{1}^{-1} C_{1} C_{2}^{-1}$ & $\frac{13}{4}=3.25$ & 7 \\
\hline$\lambda_{2}^{1-\frac{n}{2}} \lambda_{1}^{-1} C_{2} C^{-1}\left(\lambda_{1}, \lambda_{2}\right)$ & $\frac{8}{3(4-\pi)} \approx 3.11$ & $\frac{81 \sqrt{3}}{27 \sqrt{3}-8 \pi} \approx 6.49$ \\
\hline $1-\left(\lambda_{2}-\lambda_{1}\right) \lambda_{2}^{-1} C_{1} C_{2}^{-1}$ & $\frac{7}{16} \approx 0.44$ & $\frac{1}{3} \approx 0.33$ \\
\hline$\lambda_{1}^{1-\frac{n}{2}} \lambda_{2}^{-1} C_{2} C^{-1}\left(\lambda_{1}, \lambda_{2}\right)$ & $\frac{1}{3(4-\pi)} \approx 0.39$ & $\frac{3 \sqrt{3}}{27 \sqrt{3}-8 \pi} \approx 0.24$ \\
\hline
\end{tabular}

From this table it can be seen that, for $n=1$ and $\lambda_{2}=4 \lambda_{1}$ or $\lambda_{2}=9 \lambda_{1}$, Theorem 4 is better than Theorem 1. One may calculate $C\left(\lambda_{1}, \lambda_{2}\right)$ and compare the two theorems in the case $\lambda_{2}=k^{2} \lambda_{1}$ with an even larger $k$, say $k=4,5, \cdots$, which is more complicated. If $\lambda_{2} / \lambda_{1}$ is not a square power of an integer, it seems hard to calculate $C\left(\lambda_{1}, \lambda_{2}\right)$ and to compare the results of Theorems 1 and 4 . 
c) Also in the one dimensional case, if $\lambda_{2}>\lambda_{1}$, then

$$
C\left(\lambda_{1}, \lambda_{2}\right)>\frac{C_{2}}{\sqrt{\lambda_{2}}}
$$

and we have

$$
m_{1}\left(1, \lambda_{1}, \lambda_{2}\right) \leq 1+\left(\lambda_{2}-\lambda_{1}\right) \lambda_{1}^{-1} C_{1} C_{2}^{-1}=\frac{1}{4}+\frac{3 \lambda_{2}}{4 \lambda_{1}}<\frac{\lambda_{2}}{\lambda_{1}}
$$

and

$$
m_{2}\left(1, \lambda_{1}, \lambda_{2}\right) \leq \sqrt{\lambda_{1}} \lambda_{2}^{-1} C_{2} C^{-1}\left(\lambda_{1}, \lambda_{2}\right)<\sqrt{\frac{\lambda_{1}}{\lambda_{2}}}
$$

Therefore, Theorem 4 improves [22, Theorem 2(iv)] in the one dimensional case.

d) Extensions of Sirakov's results were also obtained, in particular, in the one dimensional case in 7 .

\section{ACKNOWLEDGMENT}

The authors would like to thank the referee for suggestions for improving the writing of the paper, especially in the one dimensional case.

\section{REFERENCES}

[1] N. Akhmediev and A. Ankiewicz, Partially coherent solitons on a finite background, Phys. Rev. Lett., 82 (1999), 2661-2664.

[2] A. Ambrosetti and E. Colorado, Bound and ground states of coupled nonlinear Schrödinger equations, C. R. Math. Acad. Sci. Paris, 342 (2006), 453-458. MR.2214594 (2006j:35057)

[3] A. Ambrosetti and E. Colorado, Standing waves of some coupled nonlinear Schrödinger equations, J. London Math. Soc. (2), 75 (2007), 67-82. MR2302730(2008f:35369)

[4] T. Bartsch and Z.-Q. Wang, Note on ground states of nonlinear Schrödinger systems, J. Partial Differential Equations, 19 (2006), 200-207. MR2252973(2007h:35274)

[5] D. N. Christodoulides, T. H. Coskun, M. Mitchell, and M. Segev, Theory of incoherent selffocusing in biased photorefractive media, Phys. Rev. Lett., 78 (1997), 646-649.

[6] E. N. Dancer and J.-C. Wei, Spike solutions in coupled nonlinear Schrödinger equations with attractive interaction, Trans. Amer. Math. Soc., 361 (2009), 1189-1208. MR.2457395

[7] D.G. de Figueiredo and O. Lopes, Solitary waves for some nonlinear Schrödinger systems, Ann. Inst. H. Poincaré Anal. Non Linéaire, 25 (2008), 149-161. MR2383083 (2009e:35073)

[8] B. D. Esry, C. H. Greene, J. P. Burke Jr., and J. L. Bohn, Hartree-Fock theory for double condensates, Phys. Rev. Lett., 78 (1997), 3594-3597.

[9] G. M. Genkin, Modification of superfluidity in a resonantly strongly driven Bose-Einstein condensate, Phys. Rev. A, 65 (2002), No. 035604.

[10] F. T. Hioe, Solitary waves for $N$ coupled nonlinear Schrödinger equations, Phys. Rev. Lett., 82 (1999), 1152-1155.

[11] F. T. Hioe and T. S. Salter, Special set and solutions of coupled nonlinear Schrödinger equations, J. Phys. A: Math. Gen., 35 (2002), 8913-8928. MR 1946865(2003k:35230)

[12] T. Kanna and M. Lakshmanan, Exact soliton solutions, shape changing collisions, and partially coherent solitons in coupled nonlinear Schrödinger equations, Phys. Rev. Lett., 86 (2001), 5043-5046.

[13] T.-C. Lin and J.C. Wei, Ground state of $N$ coupled nonlinear Schrödinger equations in $R^{n}$, $n \leq 3$, Comm. Math. Phys., 255 (2005), 629-653. MR2135447 (2006g:35044)

[14] T.-C. Lin and J.C. Wei, Spikes in two coupled nonlinear Schrödinger equations, Ann. Inst. H. Poincaré Anal. Non Linéaire, 22 (2005), 403-439. MR2145720 (2006a:35065)

[15] T.-C. Lin and J.C. Wei, Spikes in two-component systems of nonlinear Schrödinger equations with trapping potentials, J. Differential Equations, 229 (2006), 538-569. MR2263567 (2007h:58031)

[16] Z.L. Liu and Z.-Q. Wang, Multiple bound states of nonlinear Schrödinger systems, Comm. Math. Phys., 282 (2008), 721-731. MR2426142 
[17] Z.L. Liu and Z.-Q. Wang, Ground states and bound states of a nonlinear Schrödinger system, Adv. Nonlinear Studies, to appear.

[18] L.A. Maia, E. Montefusco, and B. Pellacci, Positive solutions for a weakly coupled nonlinear Schrödinger system, J. Differential Equations, 229 (2006), 743-767. MR2263573 (2007h:35070)

[19] E. Montefusco, B. Pellacci, and M. Squassina, Semiclassical states for weakly coupled nonlinear Schrödinger systems, J. Eur. Math. Soc., 10 (2008), 47-71. MR2349896 (2008i:35088)

[20] M. Mitchell, Z. Chen, M. Shih, and M. Segev, Self-trapping of partially spatially incoherent light, Phys. Rev. Lett., 77 (1996) 490-493.

[21] A. Pomponio, Coupled nonlinear Schrödinger systems with potentials, J. Differential Equations, 227 (2006), 258-281. MR2233961 (2007e:35263)

[22] B. Sirakov, Least energy solitary waves for a system of nonlinear Schrödinger equations in $\mathbb{R}^{n}$, Comm. Math. Phys., 271 (2007), 199-221. MR.2283958(2007k:35477)

[23] E. Timmermans, Phase separation of Bose-Einstein condensates, Phys. Rev. Lett., 81 (1998), $5718-5721$.

[24] J.C. Wei and T. Weth, Nonradial symmetric bound states for a system of two coupled Schrödinger equations, Rend. Lincei Mat. Appl., to appear.

[25] J.C. Wei and T. Weth, Radial solutions and phase separation in a system of two coupled Schrödinger equations, Arch. Rational Mech. Anal., 190 (2008), 83-106. MR2434901

(J. Chang and Z. Liu) School of Mathematical Sciences, Capital Normal University, Beijing 100048, People's Republic of China

(J. Chang) Department of Mathematics, Changzhi University, Shanxi 046011, People's Republic of China 